FERMILAB-TM-2230

January 2004

\title{
Accelerator/Experiment Operations - FY 2001 Through FY 2003
}

\author{
J. Conrad, D. Denisov, S. Holmes, M. Lindgren, W. Louis, R. Roser
}

Edited by J. Appel

This Technical Memorandum (TM) summarizes the accelerator and experiment operations for the period FY 2001 through FY 2003. The plan is to have an annual TM to gather such information in one place. In this case, the information concerns the startup of Run II at the Tevatron Collider and the beginning of the MiniBooNE neutrino experiment. While the focus is on the FY 2003 efforts, this document includes summaries of the earlier years where available for completeness.

Each section was prepared by the relevant authors, and was somewhat edited for uniformity for inclusion in this summary.

\section{Collider (Steve Holmes)}

$\underline{F Y 2002}$

The cumulative integrated luminosity for the fiscal year was $83 \mathrm{pb}^{-1}$, just above the budget performance measure for the year. By September, 2002, the typical peak luminosity was about $2.2 \times 10^{31} \mathrm{~cm}^{-2} \mathrm{~s}^{-1}$ and the typical weekly integrated luminosity was about $4 \mathrm{pb}^{-1}$. This is a factor of three to four improvement over some eight months, but it is still a factor of about two below the level set as a goal in the original plan. The unscheduled down time for the accelerator complex was $17 \%$, within the goal of $20 \%$. The cumulative store time for the collider was 2991 hours. The total up time (store hours, shot setup, and accelerator studies combined) was 5262 hours for the fiscal year.

The Tevatron Collider operation in January, 2002 was not achieving the luminosity expected. The typical peak luminosity was about $0.7 \times 10^{31} \mathrm{~cm}^{-2} \mathrm{~s}^{-1}$ and the typical weekly integrated luminosity was about $1 \mathrm{pb}^{-1}$. The Laboratory formulated a very aggressive plan for improving the luminosity by an order of magnitude over the course of the calendar year. Although this plan identified the major limits to luminosity and the nature of the corrections needed, it underestimated the time and effort needed to diagnose the detailed problems and to introduce fixes in hardware and operation.

By the end of FY 2002, performance was reliably at the highest level of the run. Store 1775 showed the highest luminosity ever in the Tevatron, $2.85 \times 10^{31}$. The subsequent two stores were also in the $2.8 \times 10^{31}$ range. The twenty-times running luminosity average hit $2.7 \times 10^{31}$. Integrated luminosity for a week reached a Run II record $4.8 \mathrm{pb}^{-1}$. Improved luminosity performance was attributed to the implementation of the Accumulator shot lattice, to enhanced Accumulator stochastic cooling, to the Tevatron pbar Beam Line Tuner (BLT) and to beam- 
loading compensation on pbar coalescing cycles. Nonetheless, we continued to experience problems executing the Main Injector to Tevatron antiproton transfer correctly and have indications of optics mismatches. A record stacking rate of $12.4 \mathrm{~mA} /$ hour was achieved.

By the end of the fiscal year, it appeared that we had the capability of producing stores of $3.0 \times 10^{31} \mathrm{~cm}^{-2} \mathrm{~s}^{-1}$ from $140 \mathrm{~mA}$ stacks. We were getting used to life with the BLT, and while its utilization improved efficiency and reduced emittance dilution, significant dilution of antiprotons on injection into the Tevatron still remained. Getting to $4 \times 10^{31}$ seemed to require doing a better job of preserving both proton and antiproton emittances throughout the complex, as well as raising the proton intensity without causing instabilities.

Proton and antiproton lifetimes at $150 \mathrm{GeV}$ remained poor $(<2$ hours). Controlling the emittances is the key to improving this. We had very good pbar emittances $(10 \pi$ at $150 \mathrm{~mA}$ stacks) through $150 \mathrm{GeV}$ in Main Injector, but were blowing up on the $\mathrm{MI} \rightarrow \mathrm{Tev}$ transfer. Blowup was reduced (but not eliminated) following implementation of the Tevatron BLT. We continued to suffer significant loss of both protons and pbars during the Tevatron acceleration ramp.

Pbar efficiency to low beta depends on stack size and proton load. Values listed here are from store 1775 which was our 2002 record store. This particular store emanated from a $135 \times 10^{10}$ stack.

$\begin{array}{ll} & \underline{(\text { Store } 1775)} \\ \text { Luminosity } & 2.85 \times 10^{31} \\ \text { Protons/bunch } & 171 \times 10^{9} \\ \text { Pbars/bunch } & 15.8 \times 10^{9} \\ \text { Pbar transmission } & 47 \% \\ \text { From stack size } & 135 \times 10^{10} \\ \text { Mean Emittance } & 16 \pi\end{array}$

Prior experience has shown pbar efficiencies up to $58 \%$ from lower $\left(80-90 \times 10^{10}\right)$ stacks.

$\begin{array}{lrr}\text { Typical transfer efficiencies (store 1750): } & \frac{\text { Pbars }}{\text { Pccumulator } \rightarrow \text { MI }} & \text { Protons } \\ \text { AI acceleration } & 100 \% & \\ \text { MI Coalescing } & 77 \% & 85 \% \\ \text { MI } \rightarrow \text { Tevatron } & 84 \% * & 89 \% \\ \text { Tevatron } 150 \mathrm{GeV} & 88 \% & 81 \% \\ \text { Tevatron acceleration } & 92 \% & 92 \% \\ \text { Low beta Squeeze+halo remove } & 90 \% & 95 \% \\ \text { TOTAL } & 47 \% & 54 \%\end{array}$

*Tevatron proton FBI was reading $15 \%$ low.

The primary issues in getting into the $3-4 \times 10^{31} \mathrm{~cm}^{-2} \mathrm{~s}^{-1}$ range appeared to be: preservation of the good antiproton emittances being generated in the Accumulator, preservation of proton 
emittances through the complex, control of instabilities in the Tevatron that were limiting proton intensities, and further improvements to the Tevatron $150 \mathrm{GeV}$ lifetime and acceleration efficiency.

At the end of FY 2002, the priorities were:

1. Transverse emittance preservation through the complex.

The Tevatron BLT was implemented (as of store 1750). Initial experience was promising. We had somewhat better transmission and lower emittances. However, the first pbar bunch (in each four-bunch train) was not being injected correctly and there was residual emittance growth, believed to be due to optics mismatch, on all bunches. Protons were to be next. We had started on an injection damper in parallel.

2. Matching optics in the A1 line.

Data were not totally consistent, but indicated $40-80 \%$ emittance growth due to optical mismatch in the transfer line.

3. Implementing dampers to stablize the proton beam in the Tevatron.

Open loop measurements looked good. Horizontal damper was in commissioning, but was creating too much noise when the loop was closed.

4. Eliminating beam loss (protons and pbars) at the start of the Tevatron acceleration ramp.

The cause was not understood, but large chromaticity was suspected.

5. Opening up the Tevatron/CZero aperture.

Studies indicated we could increase the injection helix by $\sim 30 \%$ in each plane if we could open the aperture at CZero.

6. Implementing $53 \mathrm{MHz}$ beamloading in the Main Injector.

This was done on the proton coalescing cycles, and work had begun on pbar coalescing cycles.

7. Understanding longitudinal emittances throughout the complex.

Two coupled bunch modes were identified in the Main Injector. A longitudinal damper was required to fix this.

The Main Injector was capable of providing $280 \times 10^{9}$ protons reliably with 7 -bunch coalescing. We believed that we can operate at about $200 \times 10^{9}$ protons/bunch without dampers and without blowup issues. However, instabilities, and long-range beam-beam effects on pbars, prompted us to keep protons in the $175-180 \times 10^{9}$ range. The FY 2002 milestones anticipated $250 \times 10^{9}$ protons/bunch, but required Tevatron dampers.

The record number of antiprotons in collision was $572 \times 10^{9}\left(15.9 \times 10^{9} / \mathrm{bunch}\right)$ on store 1750 (September 12). Pbar efficiency on that store was $48 \%$ and luminosity was $2.6 \times 10^{31} \mathrm{~cm}^{-2}$ $\mathrm{s}^{-1}$. This shot was the first with the Tevatron BLT implemented. It showed unusually good pbar transmission from MI-Tev for this size stack and reduced emittance dilution. 
The cumulative integrated luminosity for the fiscal year was $240 \mathrm{pb}^{-1}$, which was a factor of three beyond FY 2002 and classified as "Outstanding." The unscheduled down time for the accelerator complex was $15.5 \%$ during FY 2003, well within the goal of $20 \%$. The cumulative store time for the collider was 3556 hours. The total up time (store hours, shot setup and accelerator studies combined) was 5366 hours for the fiscal year. The peak luminosity averaged over 20 consecutive stores reached $4 \times 10^{31} \mathrm{~cm}^{-2} \mathrm{~s}^{-1}$ at times. The typical weekly integrated luminosity over the latter part of the year was $6.5 \mathrm{pb}^{-1}$.

The FY 2003 run ended at 16:00 on Sunday, September 7. The fiscal year total integrated luminosity compared favorably with the goals established at the beginning of the year (base $=200 \mathrm{pb}^{-1}$, stretch $\left.=320 \mathrm{pb}^{-1}\right)$, and to the design goal presented in the Run II Plan $\left(220 \mathrm{pb}^{-1}\right)$ - although based on two more running weeks than assumed in the Plan, weeks that will come out of FY 2004 operations. Startup of Tevatron operations in FY 2004 was scheduled for November $17,2003$.

Several configuration changes were introduced during study periods at the end of the fiscal year, including:

- New acceleration helix: The acceleration helix was adjusted by integrating additional separators to provide greater separation above $500 \mathrm{GeV}$ on the acceleration ramp (starting with store 2953). This appeared to have gained a few percent in acceleration efficiency in the Tevatron. All subsequent stores were run in this configuration and this was regarded as the operational helix for the November startup.

- $\quad 2.5 \mathrm{MHz}$ acceleration: One operational store (store 2985) was loaded utilizing $2.5 \mathrm{MHz}$ acceleration of antiprotons in the Main Injector. The goals of $2.5 \mathrm{MHz}$ acceleration were to reduce the longitudinal emittance of antiproton bunches delivered to the Tevatron and (possibly) to increase the coalescing efficiency. The attempt was mostly successful. Overall the emittances were slightly reduced and the efficiencies were only down by a few percent (due to a problem identified on the last three (of nine) antiproton shots). We expected to continue to work towards establishing $2.5 \mathrm{MHz}$ acceleration as the standard following the shutdown.

- New $150 \mathrm{GeV}$ injection optics: An adjustment to the $150 \mathrm{GeV}$ Tevatron optics was introduced on store 2988 and utilized for this and the final store. The goal was reduced emittance dilution on the Main Injector to Tevatron transfer. This appeared to have been successful - emittances measured in the Tevatron were down by $2-3 \pi$. There was still room for improvement, but we expected that following realignments in the Tevatron during the shutdown we would be starting over on this.

- New Tevatron damper power amplifier: The higher power amplifier for the horizontal damper was installed before store 2985. The purpose of the new amplifier was to provide more gain to allow damping of injection oscillations. Initial operational difficulties were encountered and as a result the dampers were turned off for stores 2985 and 2988. Difficulties were rectified during a subsequent studies period and the dampers were back on for the final store (2997), but without injection damping implemented. 
Implementation of the new vertical amplifier and injection damping in both planes will occur during the November startup.

The final store (2997) had all changes described above implemented with the exception of $2.5 \mathrm{MHz}$ acceleration. That store established the record for the best ratio of luminosity to antiproton stack size $\left(4.1 \times 10^{31} / 119 \times 10^{10}\right)$.

The luminosity performance at the end of the fiscal year was at the highest level of the run. The $20 \times$ running luminosity average reached $4.00 \times 10^{31}$ on August 22, dipping slightly to $3.90 \times 10^{31}$ at the end of the run, reflecting lower stacks as studies took away from stacking. Primary limitations at the end of the fiscal year included:

- Antiproton stack size. Typical stack sizes varied greatly as a function of stacking time. Typical goals were to shoot from $160 \times 10^{10}$. We will be striving for $190 \times 10^{10}$ stacks in FY 2004. This will require a significant (50\%) increase in the stacking rate.

- Antiproton efficiency. This was typically in the $70 \%$ range. Efficiency was stack-sizedependent, ranging from the mid $60 \%$ 's for stacks above $160 \times 10^{10}$ to the high $70 \%$ 's for stacks below $130 \times 10^{10}$. The largest loss point remained Tevatron acceleration. The goal for FY 2004 is 75\% antiproton efficiency from large stacks.

- Antiproton emittance. We had significant emittance growth on the Main Injector to Tevatron antiproton transfer for the entire run. This will continue to be a point of emphasis during FY 2004. We expect to have significantly reduced coupling in the Tevatron after the shimming of cold masses during the shutdown.

- $\mathrm{CDF}$ losses. High losses at CDF at the beginnings of stores was an intermittent problem.

Switchyard 120 was operated in commissioning mode for the first time just before the September shutdown. There was delivery of fast extracted beam to MTest (for a user!). Beam was also resonantly extracted from the Main Injector but was not visible on the first profile monitor in the beam transfer line. During day one of the shutdown it was discovered that beam had been striking the extraction Lambertson.

Status relative to the FY 2003 Plan

Performance milestones for the end of FY 2003 and actuals:

\begin{tabular}{lrrrr} 
& Base Profile & Stretch Profile & $\underline{\text { Actual }}$ \\
\cline { 2 - 4 } Median Initial Luminosity & $5.4 \times 10^{31}$ & $7.6 \times 10^{31}$ & $4.0 \times 10^{31}$ & $*$ \\
Protons/bunch & $200 \times 10^{9}$ & $215 \times 10^{9}$ & $248 \times 10^{9}$ & $*$ \\
Pbars/bunch & $27.3 \times 10^{9}$ & $35.6 \times 10^{9}$ & $22.7 \times 10^{9}$ & $*$ \\
Effective Emittance & 17.2 & 17.2 & 23.2 & $*$ \\
Hourglass Factor & 0.70 & 0.70 & 0.65 & $*$ \\
& & & & \\
FY 2003 integrated luminosity & 218.0 & 311.0 & $239.9 \mathrm{pb}^{-1}$ \\
Best week & 9.2 & 14.9 & $9.7 \mathrm{pb}^{-1}$ \\
FY 2003 integrated store hours & 2898 & 3382 & $3556 \mathrm{hours}^{-1}$
\end{tabular}

* Actuals correspond to simultaneous performance on the median store of the last two weeks. 
Status of schedule milestones:

Milestone

Date

Accumulator Return to Stacking Ramp Operational

$2 / 15 / 03$

Actual/Forecast

Complete Installation of New VFC Cards

$2 / 15 / 03$

$12 / 5 / 02$ (A)

Complete CZero Lamberson Replacement

$2 / 15 / 03$

$12 / 23 / 02(\mathrm{~A})$

Commission Tevatron Injection Dampers

$5 / 15 / 03$

$2 / 1 / 03(\mathrm{~A})$

Accumulator Bands 2 \& 3 Equalizers Commissioned

$6 / 1 / 03$

$11 / 20 / 03(\mathrm{~F})^{*}$

Commission Main Injector Longitudinal Damper

$200 \times 10^{10}$ Antiproton Stack in the Recycler

$7 / 1 / 03$

$2 / 15 / 03(\mathrm{~A})$

$6 / 15 / 03$

$11 / 20 / 03(\mathrm{~F})^{* *}$

$12 / 31 / 03(\mathrm{~F}) * * *$

* Power amplifiers were delayed at the vendor because of low priority relative to military orders. Both power amplifiers have now been delivered but one was damaged in transit and returned to the vendor. The remaining good unit was installed the week of $9 / 1$ and commissioned in the stability damping system. The remaining will be installed during the shutdown and both commissioned for injection damping during the startup.

** Hardware was ready for the last few months of the fiscal year, but required a three-day shutdown, which did not materialize. This was installed during the shutdown at the end of the fiscal year.

***The Recycler Commissioning plan was to be revised due to the continuing vacuum problems in the machine. We will be re-evaluating after the September 8-November 16 shutdown.

\section{Commentary on FY 2003 performance}

Peformance for FY 2003 was significantly improved over FY 2002. Integrated luminosity was up by a factor of three $\left(240 \mathrm{vs} .80 \mathrm{pb}^{-1}\right)$ and the $20 \times$ running luminosity average rose by about $50 \%\left(4.0 \times 10^{31}\right.$ vs. $\left.2.7 \times 10^{31}\right)$. We ended up showing better performance than anticipated on store hours and on protons/bunch. Hours benefited from improved reliability and more weeks of running than originally anticipated. Proton intensities benefited dramatically from removal of the CZero Lambertson magnets in January. Performance was below expectations on luminosity, antiprotons/bunch, and emittances. Antiproton efficiencies by the end of the year had improved to the $65-75 \%$ range. The primary intrinsic shortfall remained emittance (both transverse and longitudinal). The primary needs were to straighten out the MI to Tev transfer, emittance dilution on the ramp, and to implement Main Injector longitudinal dampers. We expected to make progress on this following the modifications to the Tevatron being undertaken during the shutdown.

\section{$\underline{\text { Shutdown Activities }}$}

A three-week shutdown started January 13, 2003. The main purpose of the shutdown was the replacement of the CZero Lambertson magnets with Main Injector dipoles. This reduced the impedance of the region, leading to an improvement in the proton beam stability, and allowing increased proton beam intensity and luminosity. The increased aperture also will allow for increased helix size. The replacement of the CZero Lambertson magnets has not yet been utilized for increasing the helix amplitude at $150 \mathrm{GeV}$. Helix modifications were made at the 
end of FY2003 on the acceleration ramp and largely involved utilizing additional separators to get the beams farther apart. Availability of adequate study time will be required before the full benefit will be had.

Other shutdown work included measurement of rolls of all the Tevatron dipole and quadrupole magnets, replacement of the worst of corroded Tevatron magnet stands (four magnets), the addition of shielding surrounding the A-side low beta quadrupoles at CDF, repair of the Tevatron cryogenic transfer line atop the berm, improved emittance diagnostics in the Tevatron, Recycler, and in the A1 and P1 lines, and Recycler vacuum work and heater tape removal. In addition, a red plastic magnet seal cap was discovered and removed from the AP1 transfer line from the Antiproton Accumulator to the Main Injector. Return to operations from the shutdown was slow, due first to the loss of a cryogenic dipole magnet and, later, the A14 spool piece.

Projects scheduled for the shutdown beginning September 8, 2003, counted on for improved luminosity performance, include:

1. FZero Lambertson: A liner to reduce the transverse impedance by shielding the laminations was installed.

2. Main Injector Longitudinal Damper: This was expected to preserve smaller longitudinal emittances both on antiproton production and proton loading cycles.

3. Tevatron Injection Dampers: Should reduce (modestly) emittance growth on the transfers.

4. Tevatron Realignment: We adjusted "smart-bolts" to counteract sinking cold masses on 106 dipole magnets in the BZero and DZero regions. Modeling indicated this would allow reduced skew quadrupole circuits by about a factor of three. Magnets with significant rolls were unrolled in those houses that were warmed up ( $\sim 4$ houses out of 24). Corroded magnet stands were replaced.

The figures below give some statistics on Tevatron Collider operation during the current run, which started in April, 2001. 


\section{Collider Run IIA Hours}

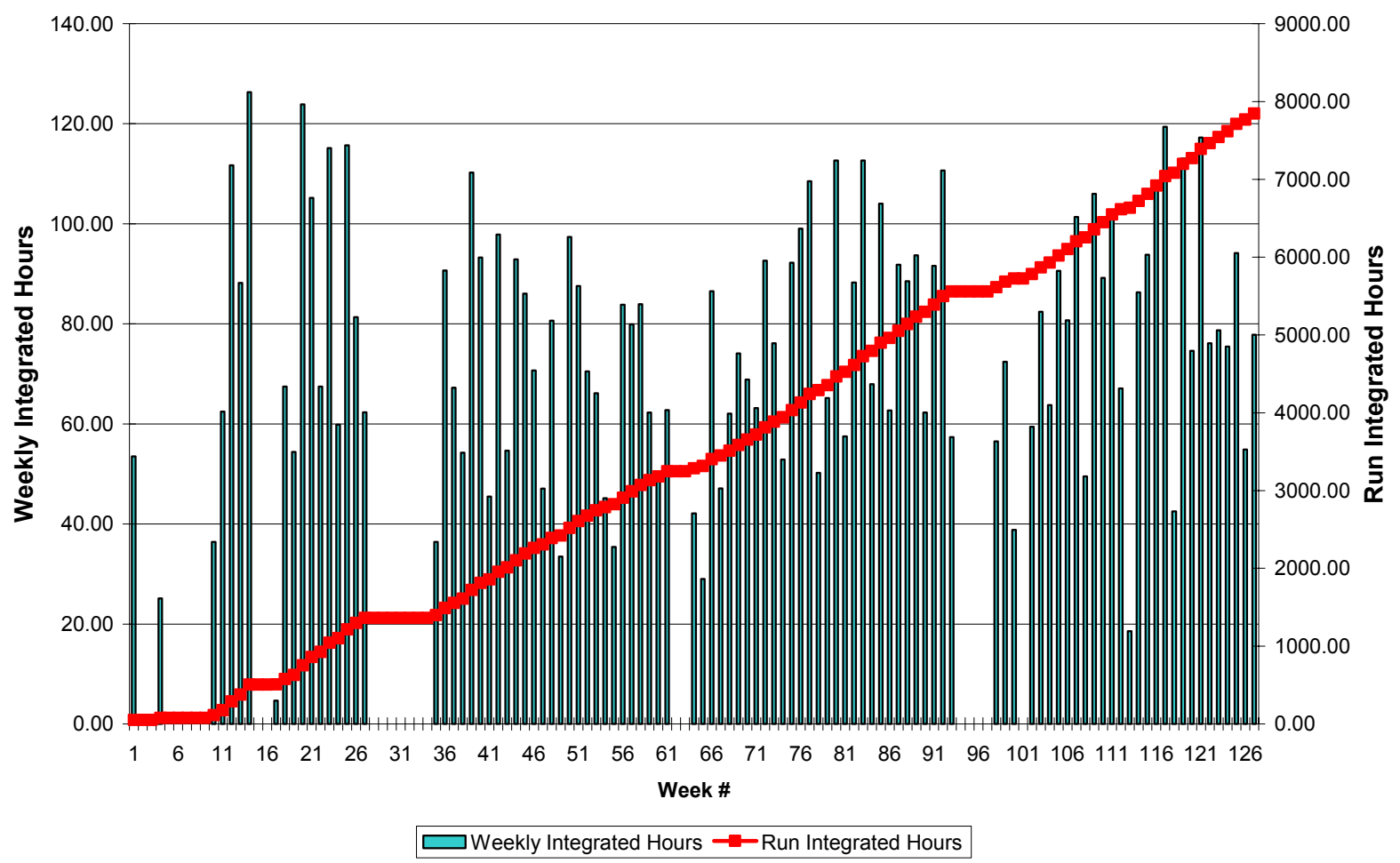

Figure 1: Hours of Tevatron Collider operation during the current running period, which started in 2001. 


\section{Collider Run IIA Luminosity}

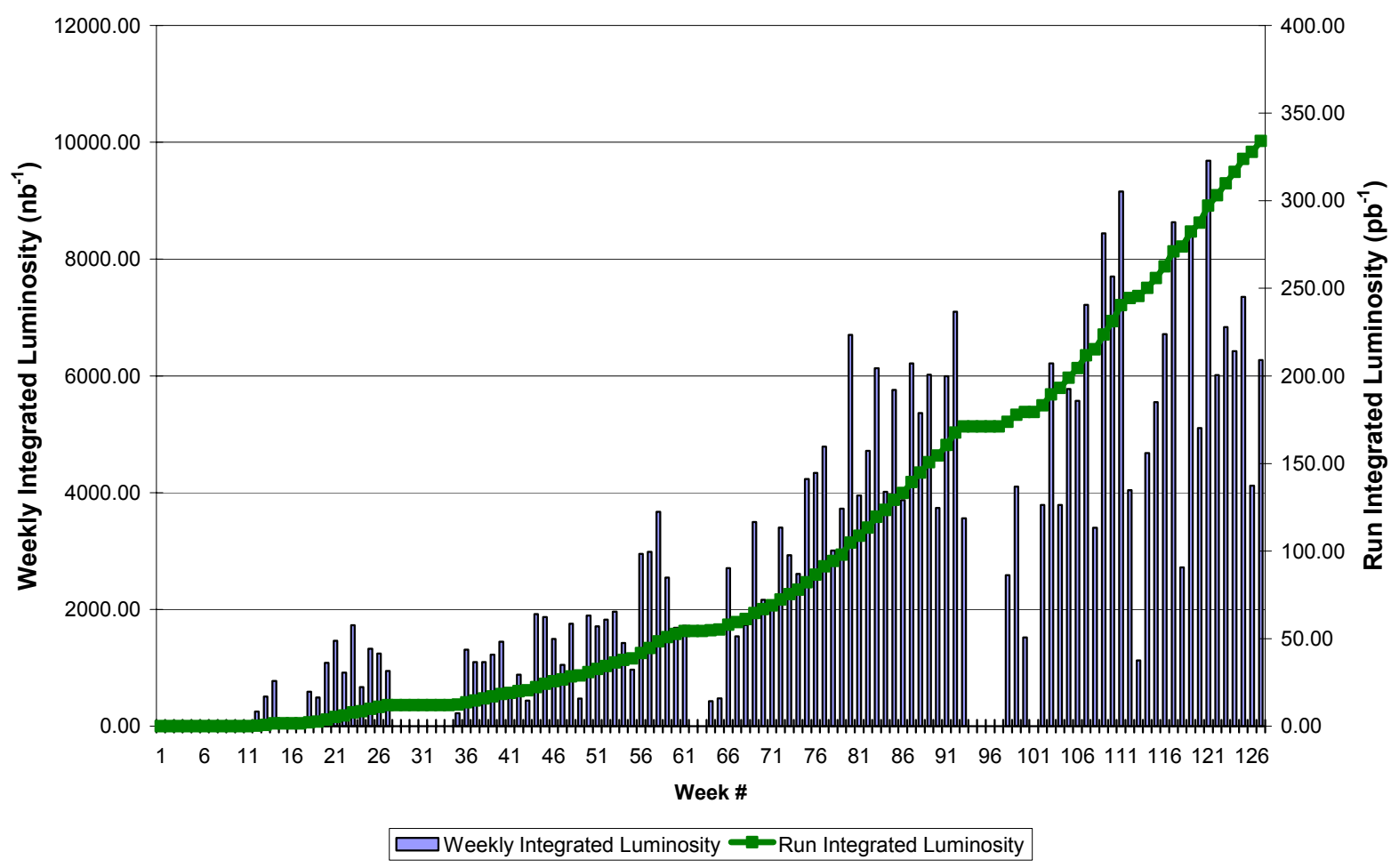

Figure 2: Tevatron Collider luminosity during the current running period, which started in 2001. 


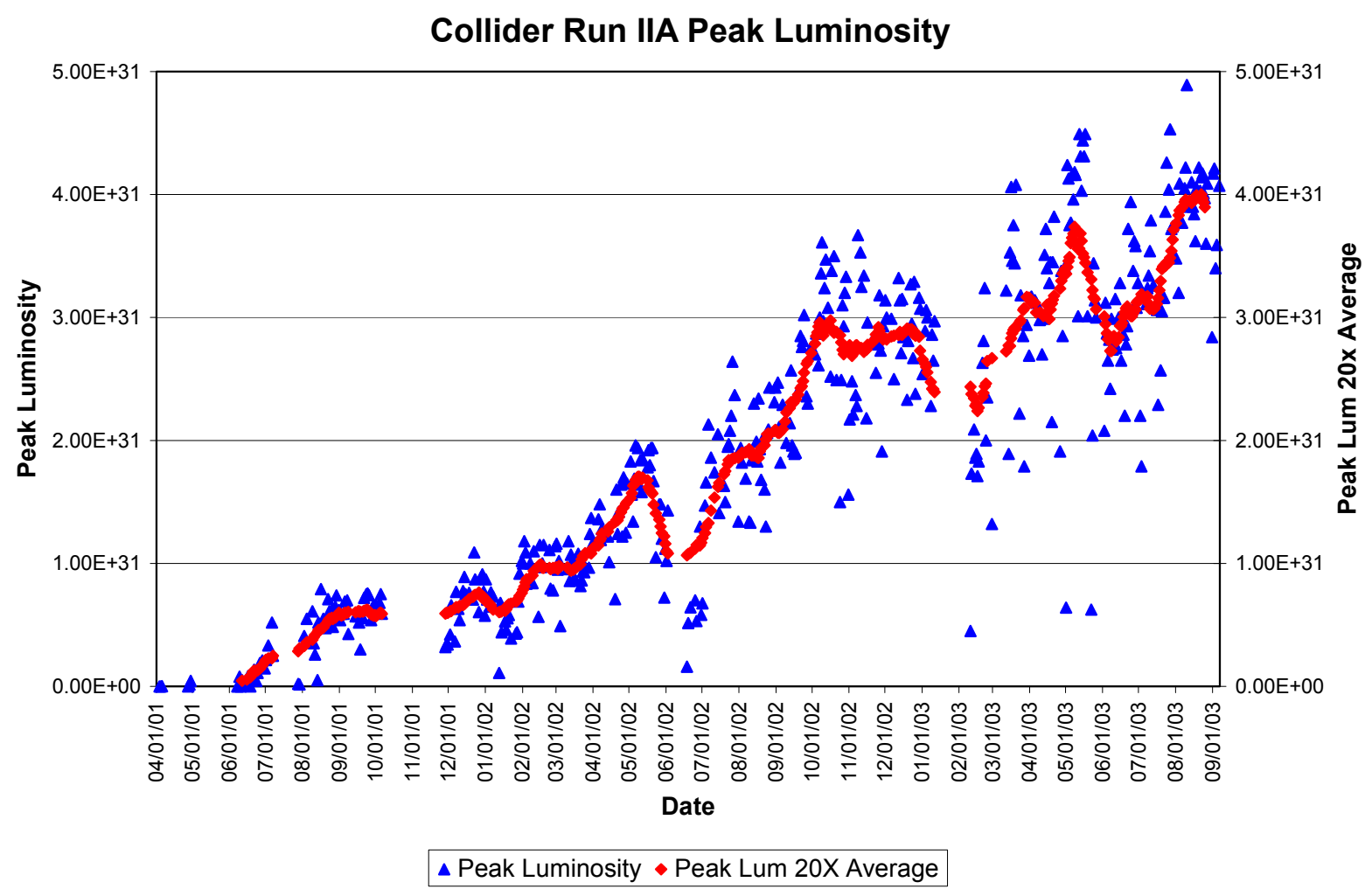

Figure 3: Peak luminosity of stores during Tevatron Collider operation for the current running period, which started in 2001.

Collider Detector at Fermilab (CDF) (Mike Lindgren and Robert Roser)

\section{$\underline{\text { Introduction }}$}

The Collider Detector at Fermilab (CDF) is a 5000-ton magnetic detector built to study $2 \mathrm{TeV}$ proton-antiproton collisions at the Fermilab Tevatron. Event analysis is based on a charged-particle tracking, magnetic momentum analysis and fine-grained calorimetry. The detector first saw collisions in 1987-88 during its engineering run. The first significant data run occurred in 1992-1996 where the detector collected $\sim 100 \mathrm{pb}^{-1}$ of data to tape and discovered the top quark. Since the detector is a multipurpose device, the collaboration was able to make significant measurements in QCD and electroweak physics as well as b-physics and exotic searches. In 1997, CDF began an aggressive upgrade program so that the experiment could make the best use of the substantial increases in luminosity expected as a result of upgrades to the pbar source and the addition of the Main Injector and Recycler into the accelerator complex. In this four-year upgrade, CDF replaced all of its tracking chambers, readout and trigger electronics, data acquisition and trigger system and improved the muon coverage. The upgraded detector was rolled into the collision hall for the start of Run II in March 2001. 
The next few pages summarize the detector operation, its major successes as well as its major problems as a function of Fiscal Year.

$\underline{F Y 2001}$

The CDF collaboration spent the first half of the year pulling the detector out of the collision hall after its "commissioning" run and focused its efforts on final silicon integration. On January 23, 2001, the central detector was rolled from the assembly hall into the collision hall in preparation for data taking. With the detector installed, the next six weeks prior to beam was used to complete cabling, water, cryogenic and gas hook-ups and the start of full detector integration testing. On March $8^{\text {th }}$, beam started to circulate in the Tevatron and the CDF detector was not fully ready. The silicon detectors were not fully cabled and while individual components had been tested, this detector had yet to be powered in its entirety. CDF spent the remainder of this fiscal year commissioning its detectors, aligning them with beam, and beginning to understand their performance.

During this commissioning phase, CDF found that $\sim 50 \%$ of the ISL (intermediate silicon layers) could not be used due to a cooling problem. An aggressive R\&D program was started to identify the cause and find a solution. By the end of FY 2002, the problem had been located the cooling lines were blocked by excess epoxy used to connect the cooling lines together.

Throughout this period, numerous accesses into the collision hall were made to replace electronics boards, complete cabling, and looking for sources of electronics noise. CDF wrote $20 \mathrm{pb}^{-1}$ of data to tape during this period. This data was very useful for commissioning the detector - but none of it will be used for final physics analysis.

$\underline{F Y 2002}$

CDF's goal in FY 2002 was to complete the commissioning of its individual detectors and trigger systems and begin to take "physics" quality data. Once this process was complete, the operations team was to turn its attention to reliability and data taking efficiency issues. Unfortunately, life wasn't so kind to us. In mid-November, scarcely one month into the new fiscal year, CDF experienced a significant beam incident. Single-turn protons continuously collided with our beam pipe on the "B-side" of the CDF detector just upstream of Q4 during accelerator start-up. That incident permanently damaged $2 / 3$ of the power supplies for the Central Outer Tracker (COT) and Silicon VME crates. These supplies are used to power the readout electronics for those detectors. Their death was the result of a high instantaneous radiation dose. Prior to that point, several of these power supplies had failed - but their failure was attributed to infant mortality. After this incident, it was clear that these supplies had a design flaw that had to be corrected and that CDF had to take significant measures to keep beam losses in its collision hall to a minimum.

CDF began a two-pronged $R \& D$ effort to address this radiation incident. It worked with the power supply manufacturer to identify the part of the power supply circuitry that was susceptible to radiation (high instantaneous current), and worked with the Beams Division modelers to design shielding which could be installed around the low-beta quad magnets 
(without rolling out the central detector), and which would substantially reduce the losses seen by the detector as a result of beam halo. After substantial effort, the power supplies were modified to be more "rad-hard" and a shielding plan was devised that could be implemented during the FY 2002 summer shutdown.

In spite of the above, CDF experienced many successes in FY 2002. On February $8^{\text {th }}$, the detector was declared ready for physics. From that point forward, all data written to tape is intended for use in physics analysis. Layer 00 (the layer of silicon mounted directly to the beam pipe) was commissioned and in April, the operation of the silicon detectors was turned over to the shift crews and was no longer under expert-only control. The level 2 trigger systems were working well and the operations staff worked on data-taking efficiency. A trigger inhibit system was built; the software to turn on detector high-voltage power supplies after beam scraping was rewritten and substantially sped up; and a variety of other improvements were made. Finally, during the June shutdown, 10 of the 12 clogged ISL cooling lines were opened using a highpowered laser and 132 of the 136 detectors in that region were able to operate. In FY 2002, CDF collected $47 \mathrm{pb}^{-1}$ of data to tape. Approximately $70 \%$ of that is useful for physics analysis with an average data-taking efficiency of $65 \%$.

\section{FY 2003}

Similar to FY 2002, CDF was off to a rocky start in FY 2003. CDF experienced a number of sudden and unexpected terminations of stores that resulted in high losses in the CDF detector. This was caused by a number of problems, most notably the loss of Tevatron RF and a "prefiring" of the Tevatron abort kicker magnets. To date, there have been five separate inadvertent terminations of stores that have resulted in as much as $200 \mathrm{rad}$ (from a single incident) at the silicon detector. In total, nine silicon ladders have been permanently damaged in two separate such incidents. In a joint partnership with the Beams Division, CDF worked on solutions to minimize the impact of these unexpected beam terminations on the silicon detectors. As an example, collimators at A11 and A48 were installed to scrape away beam, say from a kicker prefire, prior to its reaching and potentially damaging the CDF silicon detector. Furthermore, the CDF silicon experts are continually trying to reconfigure the way this detector is run to make it less susceptible to permanent damage when an incident does occur.

Throughout FY 2003, CDF improved the operation of its detector. Typically, 93\% of the silicon detector was working routinely during all stores. Dynamic prescaling of triggers was implemented to accommodate higher luminosities without incurring substantial dead time. Trigger algorithms were improved to get higher purity and thus make better use of the overall DAQ bandwidth. Operationally, a myriad of small improvements were made such that by the end of the fiscal year, CDF was routinely recording data for individual stores with greater than $90 \%$ efficiency. Furthermore, detector reliability improved substantially. The number of access requests dropped substantially and CDF was happy with $\sim 1-2$ hour/week for routine maintenance issues.

Beam-related losses remain an outstanding issue for CDF. Data-taking efficiency could be higher if accelerator losses at the beginning of a store were better understood and under control. For $\sim 80 \%$ of the stores, losses were acceptable and the silicon detectors were integrated 
immediately. However, for the remaining stores, CDF has had to wait as much as several hours before it can turn its silicon detectors on and integrate data with the entire system.

In FY 2003, there were two substantial shutdowns - three weeks in January and ten weeks beginning in early September. In the January shutdown, CDF installed its shielding on the "A-side" of the collision hall low-beta quad magnets. It also opened one of the two remaining ISL cooling lines with the laser. Since the CDF detector had been operating extremely well throughout much of FY 2003, much of the focus of the September shutdown was on reliability issues. Fans were replaced, filters changed, power supplies replaced, etc., in an effort to prevent failures once beam comes back. The shielding on the "B-side" low-beta quad magnets will be installed in October, which will complete this shielding project. In addition, two COT wire planes were replaced in situ using a custom-stringing engine. In the future, if a COT wire breaks while taking data, CDF now has confidence that it can fix the problem efficiently while the "clock is running". That procedure went very well, and the apparatus built to do this worked as planned.

In summary, CDF had a very good FY 2003. From Figure 4, you can see that CDF wrote $198 \mathrm{pb}^{-1}$ of data to tape and that $\sim 84 \%$ of it was physics quality. The average data-taking efficiency for the fiscal year was $86 \%$ and, as stated earlier, in the latter stages of FY $2003>90 \%$ data-taking efficiency was routine. 


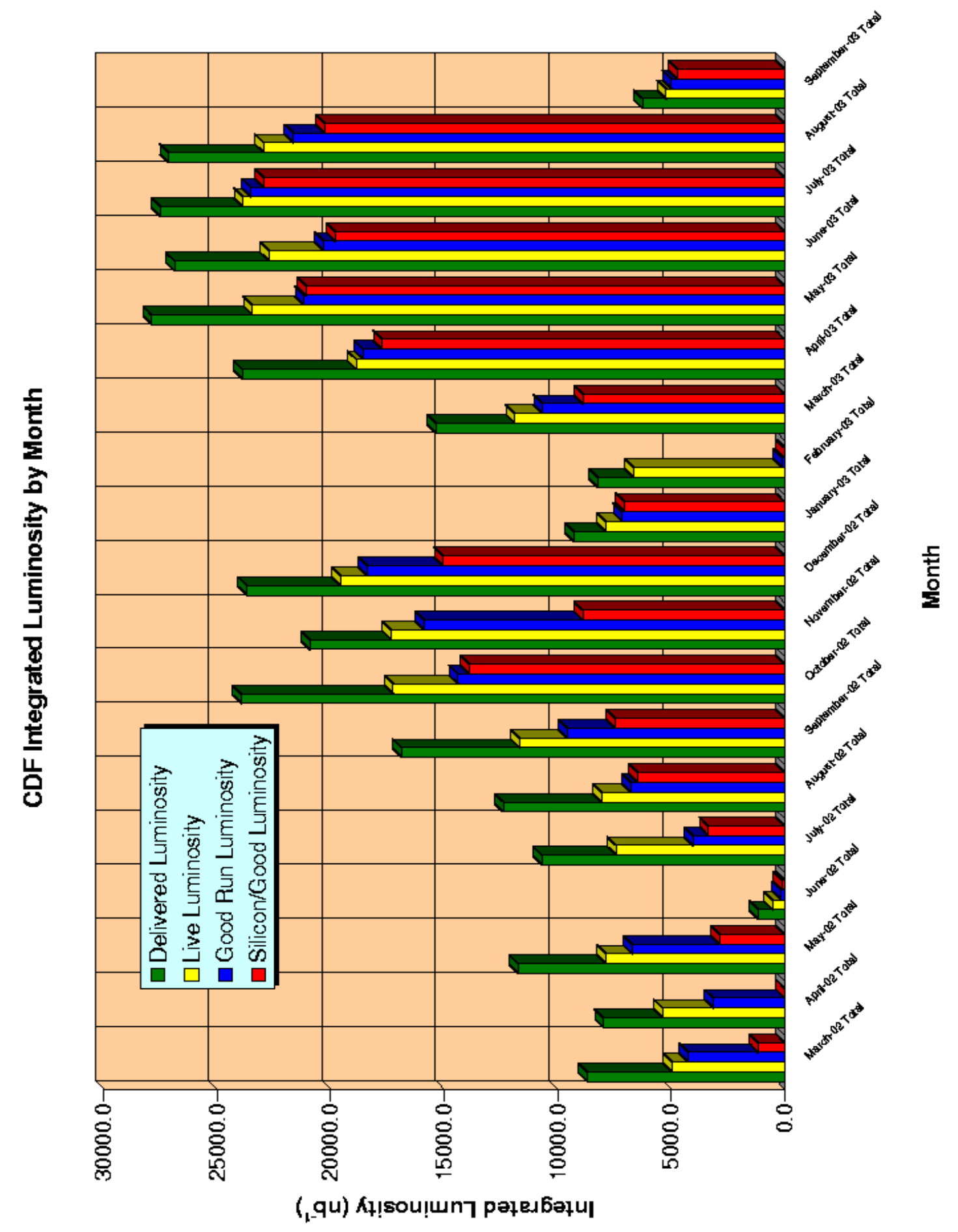

Figure 4: Monthly History of CDF luminosity since data was declared ready for physics. 


\section{$\underline{\text { DZero (D0) }}$ (Dmitri Denisov)}

This document summarizes major accomplishments in data-taking by the DZero Collaboration over the period April 2002 to September 2003. The beginning of this period is selected based on its correspondence to the full installation of front-end electronics for the DZero central fiber tracker. Total integrated luminosity collected before that date was $34 \mathrm{pb}^{-1}$ and was mainly used for commissioning of the detector, trigger, DAQ, and off-line reconstruction and analysis software. The end of the period under consideration coincides with the extended shutdown started early in September 2003, and end of FY 2003.

Completion of the fiber tracker commissioning separates Run II DZero data-taking into two distinct periods: commissioning and physics data-taking. With such a complex detector as DZero, it is practically impossible to accomplish such a transition in a short period of time. So, over the last 1.5 years, our major goal was to improve DZero data-taking efficiency as well as the quality of the physics data we are collecting. DZero defines "efficiency" of the detector operation as the ratio between luminosity DZero recorded to tapes and the luminosity the Tevatron delivered to the DZero interaction region. Periods of detector and DAQ down-time with inability to collect data reduce operating efficiency.

The monthly plot of the DZero data-taking efficiency since April 2002 is presented in Figure 5.

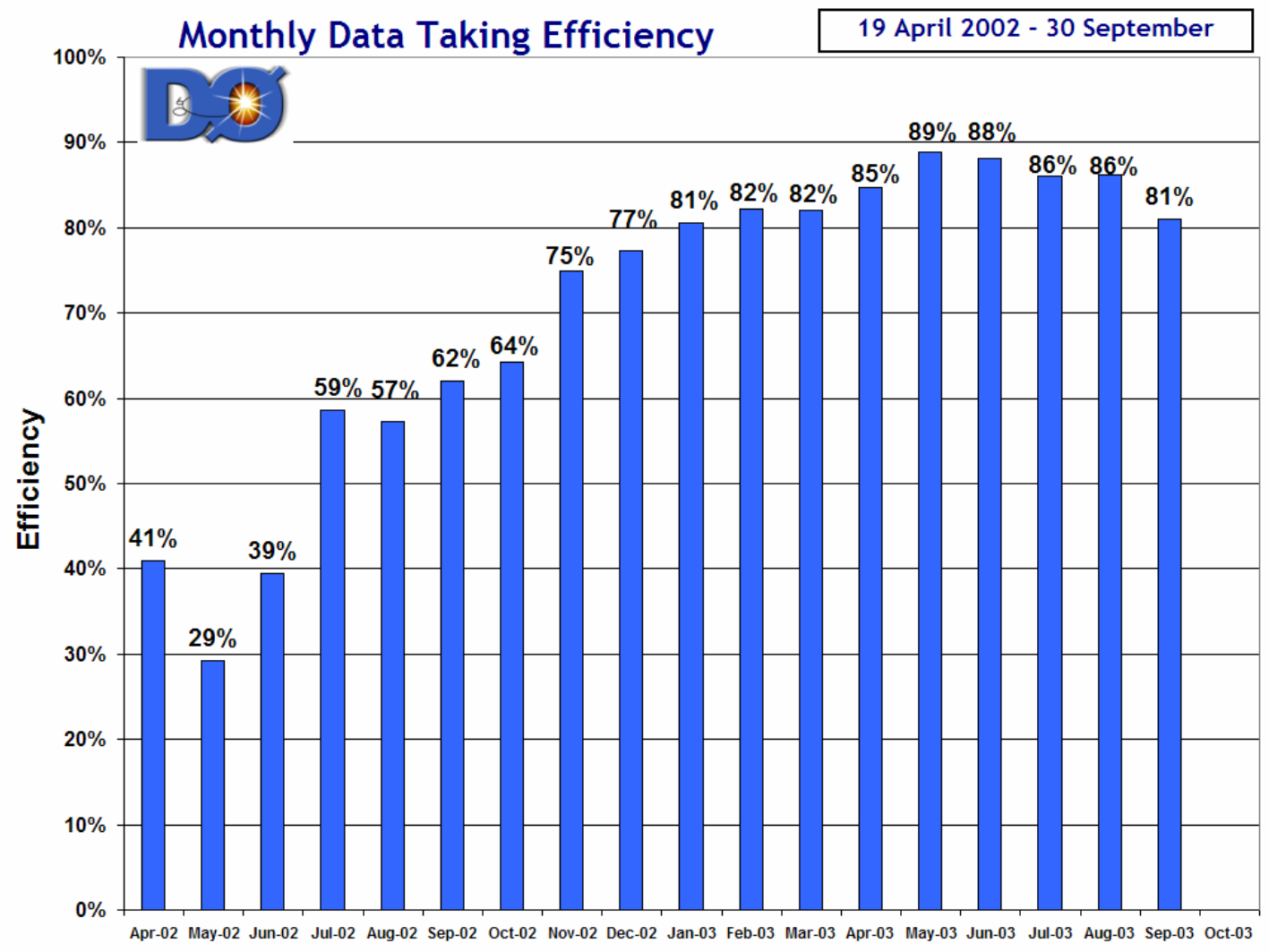

Figure 5. DZero monthly data-taking efficiency from April 2002 to September 2003. 
As is clear from Figure 5, during 2002 DZero's efforts concentrated on the steady increase of the DZero data-taking efficiency from $\sim 30 \%$ to $\sim 90 \%$. In order to achieve high operational efficiency, a large number of issues had to be resolved. Reliability of different detector systems was improved by replacement of unreliable components and changes in the operating conditions. Procedures for the shifters were developed with the goal of resolving problems quickly. DAQ system software was debugged and special software capable of automatically detecting and resolving known problems was developed. As a result, since early 2003 , DZero reached data-taking efficiency of $87 \pm 2 \%$, which is a typical number for large highenergy-physics experiments.

Before the beginning of the September 2003 shutdown, the following sources contributed to the DZero data-taking inefficiency:

1. Front-end busy in the tracking readout crates: $\sim 4 \%$.

2. Failures of detector, trigger, and DAQ components: $\sim 7 \%$.

3. Pauses in data taking at the beginning/end of the Tevatron stores, at the begin/end runs, etc.: $\sim 2 \%$.

We see no reason to expect any considerable (beyond a few percent) increase in datataking efficiency in comparison with that already achieved. Item 1 is driven by the design of the SVX II chip and can't be reduced. Analysis of incidents in Item 2 found that failures are distributed more or less randomly over wide range of the systems. Reduction in the number of failures will require considerable financial and manpower efforts, including re-design of some major elements. We are working on improvements in Item 3, but it is difficult to expect reduction beyond about $1-1.5 \%$ in this parameter. The experiment has to change prescalers every few hours, ramp high voltage up and down at the beginning and end of the stores, etc. In summary, DZero's plan is to keep physics data-taking efficiency at about the $90 \%$ level for the duration of Run II.

As a result of the improvements in the data-taking efficiency, by September 2003 the total luminosity DZero recorded to tapes was $214 \mathrm{pb}^{-1}$ or almost twice the amount of the Run I data sample; see Figure 6.

In addition to the amount of data the experiment wrote to tape, the quality of the data recorded is important as well. For example, for a few week periods in August 2002 and December 2002, two major sub-systems of the DZero detector calorimeter and muon had timing problems which made data collected by these detectors unusable for most physics studies. It took a large amount of effort to develop appropriate monitoring tools, as well as to introduce the "Global Monitor" shift. As a result, DZero had no undetected problems with the detectors during the rest of FY 2003. 


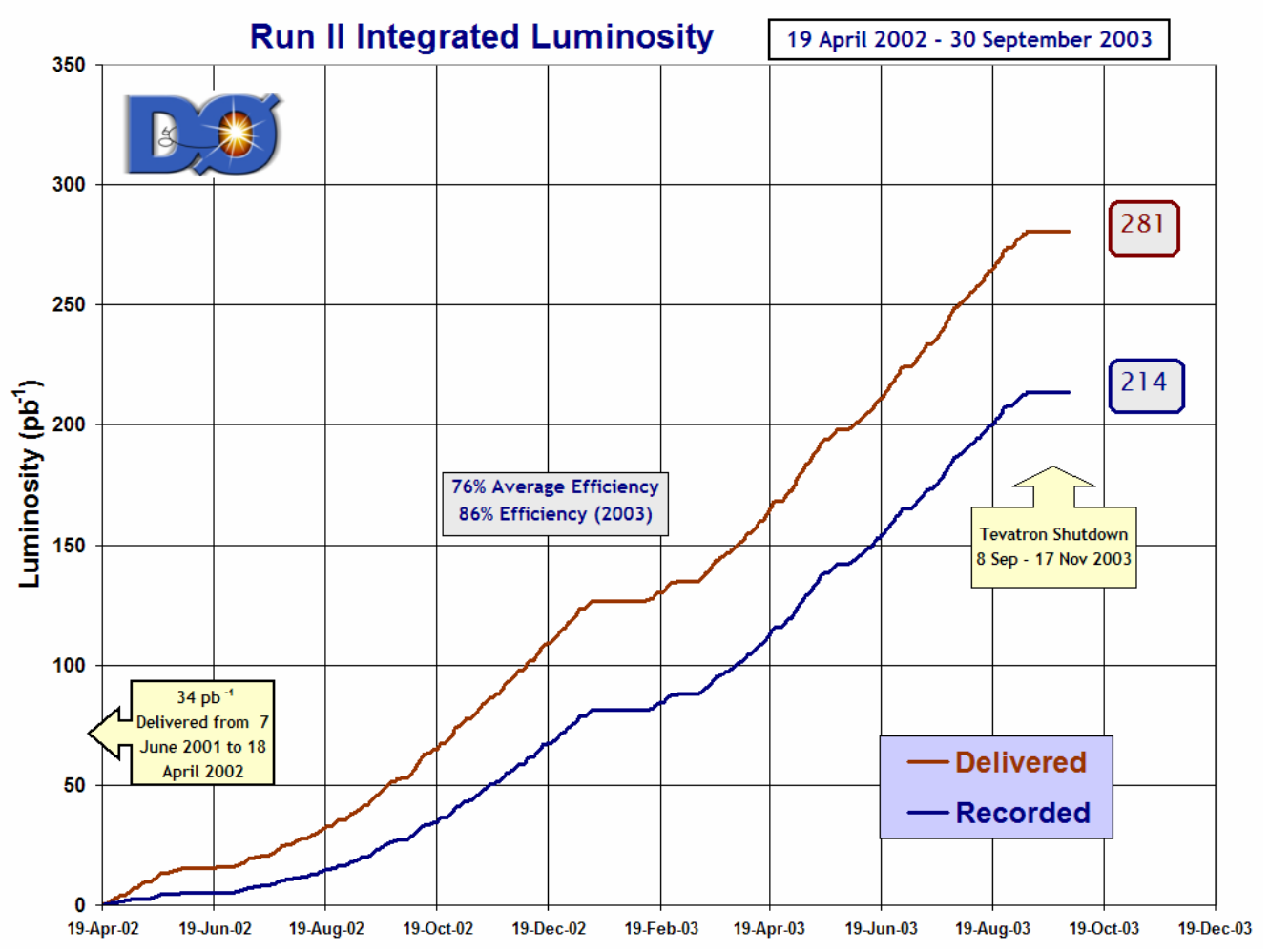

Figure 6. Delivered and integrated luminosity at DZero from April 2002 to September 2003.

Since April 2003, improvements in different sub-systems were developed and implemented. The central and forward pre-shower detectors were commissioned. The forward proton detector started to produce physics data. The Level 2 and Level 3 trigger algorithms were slightly improved, and a new Level 2 trigger hardware version ("Beta") was successfully commissioned. Calorimeter triggering was improved, and a fiber tracker trigger was commissioned.

As with any large high-energy physics detector, there is a continuous process of addition of new capabilities and improvements. In the next few months DZero expects full integration of the forward proton detector into the data and analysis streams, commissioning of the trackerbased displaced vertex trigger, as well as multiple improvements in almost every DZero subsystem.

It is important to mention that operations during the reporting period were performed safely, with no accidents involving personnel or detector components. This is due to efforts by everybody involved in the experiment operation, as well as constant efforts by the experiment management.

Over the last 1.5 years, the DZero detector moved from commissioning to stable physics data-taking. The record data set collected at the world's highest energy collider is now being used for physics analysis. 
MiniBooNE (Janet Conrad and William Louis)

\section{$\underline{\text { Introduction }}$}

The MiniBooNE experiment began taking data at Fermilab in late August, 2002, and has collected over $160 \mathrm{~K}$ total neutrino events after the first year of operation. This report summarizes the MiniBooNE construction, installation, and data collection during the fiscal years from 2001 through 2003.

$\underline{\text { FY } 2001}$

The 2001 fiscal year began with the completion of the detector civil construction and ended with the completion of the detector installation. Beneficial occupancy of the detector enclosure occurred in November, 2000, and was followed by the start of phototube installation in January, 2001. The phototube installation was completed in September, 2001. Figure 7 shows a photograph of the inside of the MiniBooNE detector tank just before the tank was sealed. Great progress was also made during this fiscal year with the beamline civil construction, which began in July, 2000, and with the construction of the magnetic focusing horn, for which testing began in July, 2001. By the end of FY 2001, the detector was complete except for oil filling.

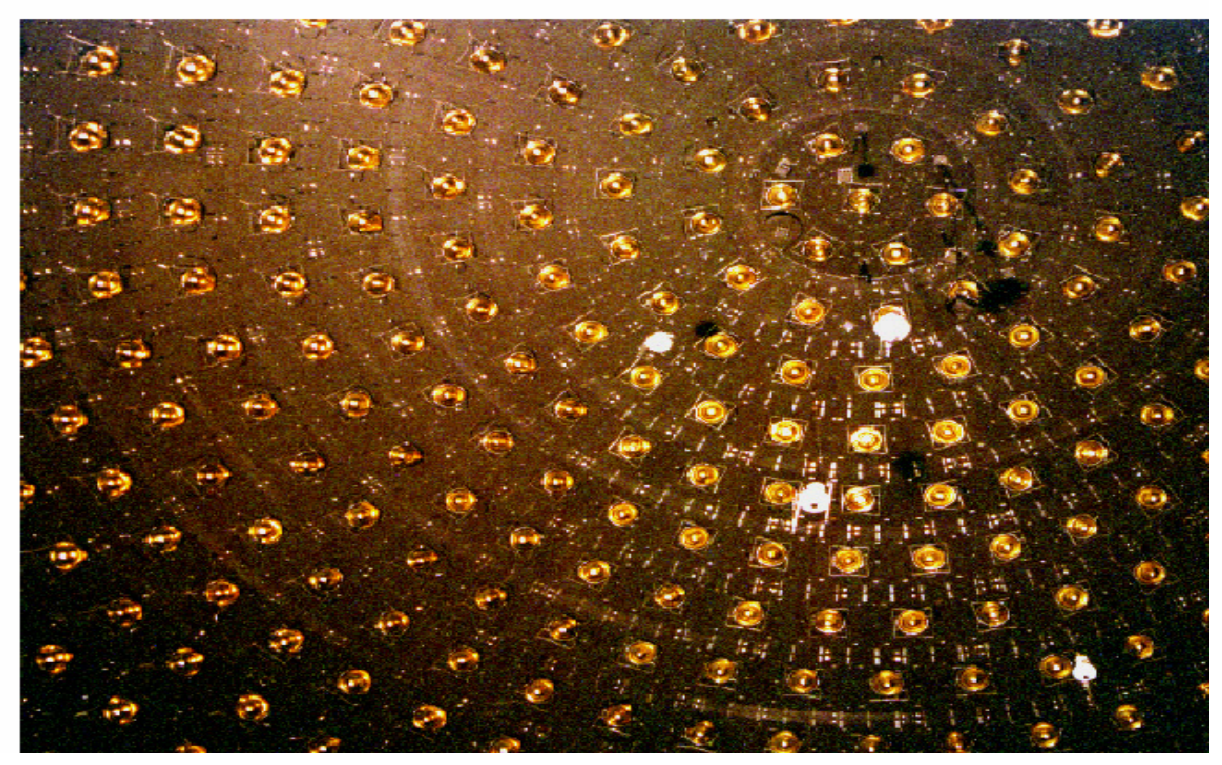

Figure 7. A photograph of the inside of the MiniBooNE detector tank just before the tank was sealed.

\section{$\underline{F Y 2002}$}

Oil filling of the detector tank began in December, 2001, and ended in May, 2002, marking the completion of the detector. With the detector fully operational, cosmic-ray data taking then ensued with the shakedown of the electronics and data acquisition system. By July, 2002, the detector was smoothly collecting cosmic-muon data. FY 2002 also marked the completion of the MiniBooNE beamline. The beamline civil construction ended in April, 2002, 
and was followed by first protons down the beamline later that month and first beam to the target position in June, 2002. Finally, first beam on target was delivered in August, 2002, which allowed MiniBooNE beam data-taking to begin.

$\underline{F Y 2003}$

The 2003 fiscal year began just after the start of MiniBooNE data-taking. Initially, the proton intensity was about an order of magnitude below the goal of $9 \times 10^{16} \mathrm{POT} / \mathrm{h}$; however, as the year evolved, the proton intensity increased to within a factor of two of the goal intensity. For example, $5.19 \times 10^{16}$ POT were delivered in an hour on May 7, 2003, and $9.6 \times 10^{18}$ POT were delivered for the day May 14, 2003. Figure 8 shows the progress during fiscal year 2003 in delivering beam to MiniBooNE. For this first year of data-taking, MiniBooNE recorded 40.54 $\mathrm{M}$ total horn pulses, $1.48 \times 10^{20}$ POT (about $30 \%$ of the yearly goal), and $162 \mathrm{~K}$ neutrino events of all types. In addition, the MiniBooNE detector and beamline performed well. For the year, about $99 \%$ of the phototube channels worked well, and the data acquisition livetime averaged $\sim 99 \%$. Furthermore, the reconstructed time, position, energy, and angular resolutions were all in agreement with expectations, and the experiment observed both CC-muon events and NC-pion events.

\section{Conclusion}

Overall, 2003 was an outstanding first year of data-taking for the MiniBooNE experiment. The detector and beamline performed well, and the Booster delivered $1.5 \times 10^{20}$ POT (30\% of the yearly goal) to the experiment. With the Booster improvements made during the 2003 shutdown, 2004 should be even better. 


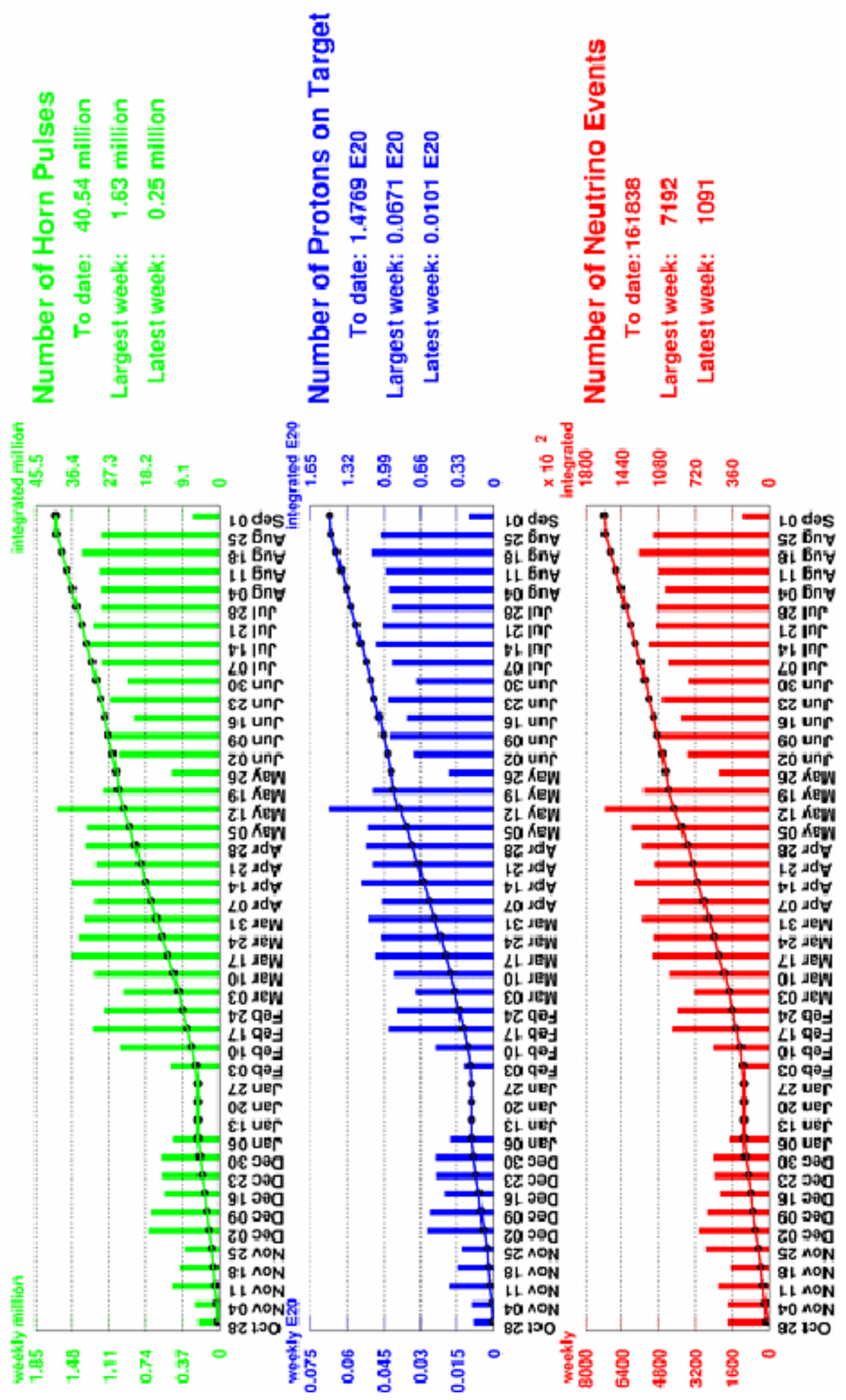

Figure 8. The progress during fiscal year 2003 in delivering beam to MiniBooNE. 